\title{
Análise de Frequência Regional de Ventos Extremos no Paraná
}

\author{
Rafael Cabral Gonçalves ${ }^{1}$ (D), Alexandre Kolodynskie Guetter ${ }^{2}$ \\ ${ }^{1}$ Programa de Pós-Graduação em Engenharia de Recursos Hídricos e Ambiental, \\ Universidade Federal do Paraná, Curitiba, PR, Brasil. \\ ${ }^{2}$ Departamento de Hidráulica e Saneamento, Universidade Federal do Paraná, \\ Curitiba, PR, Brasil.
}

Recebido em: 14 de Setembro de 2016 - Aceito em: 23 de Agosto de 2018

\begin{abstract}
Resumo
Para estimar a magnitude e frequência dos vendavais no Paraná foram analisadas as rajadas de 26 estações meteorológicas. Integrou-se os procedimentos de identificação de regiões homogêneas, utilizando o método dos componentes principais, e de análise de frequência regional, pois as séries disponíveis são muito curtas para aplicação dos métodos convencionais. Diagnosticou-se cinco regiões homogêneas: Litoral, Centro, Meio-Oeste, Oeste e Norte. Esses resultados produziram coerência geográfica, pois as estações agrupadas são vizinhas entre si e estão em locais com relevo e continentalidade semelhantes. A distribuição mais adequada para a análise de frequência regional foi a de Wakeby, cujos quantis regionais estimados exibiram elevada coerência com as diferenças espaciais diagnosticadas na estatística descritiva. Para o tempo de recorrência de 50 anos a região composta pelas estações de Cascavel e Toledo apresentou os ventos mais severos, com velocidades de 41,9 e $44,8 \mathrm{~ms}^{-1}$, respectivamente. As demais regiões produziram ventos de 35,3 a $39,7 \mathrm{~ms}^{-1}$ no Oeste, 28,8 a 38,2 $\mathrm{ms}^{-1}$ na região Central, 26,4 a 36,6 $\mathrm{ms}^{-1}$ no Norte e 21,4 a 30,6 ms ${ }^{-1}$ no Litoral. Os resultados obtidos são inovadores pois se estimaram probabilidades de ocorrências de ventos extremos usando séries de observações muito curtas, embora de boa qualidade e resolução temporal.
\end{abstract}

Palavras-chave: análise de frequência, vento extremo, rajada.

\section{Regional Frequency Analysis of Extreme Winds at Parana State, Brazil}

\begin{abstract}
Gusts from 26 meteorological stations at Paraná (Brazil) were analyzed to estimate their magnitude and frequency. This analysis integrated the homogeneous regions identification, using the principal components analysis, and regional frequency analysis, since the available dataset were too short to apply conventional methods. Similar data patterns were identified in five regions: Coast, Central, Mid-West, West and North. This results were geographical consistent, because the stations are at contiguous sites and located at regions with similar characteristics of relief and coast distance. The Wakeby distribution was selected as the most adequate for the regional frequency analysis. The regional quantiles estimated by the adjusted distribution were coherent with the spatial differences verified in the descriptive statistic. For a given return period of 50 years, the region composed by Cascavel and Toledo stations showed the most severe winds, with 41,9 and $44,8 \mathrm{~ms}^{-1}$, respectively. In the West winds vary from 35,3 to $39,7 \mathrm{~ms}^{-1}$, in the Central from 28,8 to $38,2 \mathrm{~ms}^{-1}$, in the North from 26,4 to $36,6 \mathrm{~ms}^{-1}$ and in the Coast from 21,4 to $30,6 \mathrm{~ms}^{-1}$. The results of this study are innovator since the wind probabilities were estimated using a short dataset, however with good quality and temporal resolution.
\end{abstract}

Keywords: frequency analysis, severe wind, gust.

\section{Introdução}

Deslocamentos violentos de massas de ar são um dos eventos naturais mais devastadores em habitats humanos. Devido aos danos aos habitats humanos e à infra-estrutura causados por vendavais, análises de extremos de vento formam uma parcela essencial do processo de integração entre programas governamentais contra desastres naturais e objetivos gerais de desenvolvimento.

Autor de correspondência: Rafael Cabral Gonçalves, rafael_cabral_goncalves@yahoo.com.br. 
O objetivo deste estudo foi diagnosticar a aplicabilidade de métodos para estimar a magnitude e a frequência de ventos extremos no Estado do Paraná, com o uso de séries curtas de dados. Para atingir este objetivo aplicou-se critérios de consistência para a produção de uma base de dados de vendavais, selecionou-se o método de análise de extremos mais adequado para avaliar a severidade e a frequência dos ventos e produziu-se mapa temático que apresenta os resultados da análise de extremos.

\section{Material e Métodos}

Os dados de vento usados na análise de extremos deste estudo compreendem período de 08 anos, a partir de 1998 e são provenientes de 26 estações meteorológicas operadas pelo SIMEPAR no Paraná, cujas localizações estão ilustradas na Fig. 1.

A rede de estações meteorológicas possui densidade e distribuição espacial adequadas para a cobertura da área do Estado do Paraná $\left(199.709 \mathrm{~km}^{2}\right)$ e suas distintas condições de relevo. A densidade média das estações é de uma a cada $6241 \mathrm{~km}^{2}$, sendo a distância média entre as estações da ordem de $52 \mathrm{~km}$.

Os dados de vento medidos nas estações meteorológicas do SIMEPAR são amostrados com anemômetro modelo YOUNG 05103-11A, cujas características estão sumarizadas na Tabela 1. Dentre os diversos tipos de dados eólicos disponíveis no banco de dados deste instituto, o mais adequado para análise de extremos é o maior valor horário das 60 médias dos registros medidos nos 10
Tabela 1 - Especificações dos sensores de vento instalados nas estações meteorológicas do SIMEPAR.

\begin{tabular}{lc}
\hline Parâmetro & Especificação \\
\hline Modelo & $05103-11^{\mathrm{A}}:$ YOUNG \\
$\begin{array}{l}\text { Banda de medição } \\
\text { Rajada máxima (sem danificar o } \\
\text { equipamento) }\end{array}$ & $0,0 \mathrm{~m} \mathrm{~s}^{-1}$ a $60 \mathrm{~m} \mathrm{~s}^{-1}$ \\
Erro & $100 \mathrm{~m} \mathrm{~s}^{-1}$ \\
$\begin{array}{l}\text { Velocidade mínima para medição da } \\
\text { direção }\end{array}$ & $0,3 \mathrm{~m} \mathrm{~s}^{-1}$ \\
\hline
\end{tabular}

últimos segundos de cada minuto, conforme ilustrado na Fig. 2.

Foram aplicados algoritmos de controle de qualidade dos dados cuja metodologia foi extraída de Shafer et al. (2000).

Uma vez que as séries de utilizadas são de 8 anos de extensão, utilizou-se uma estratégia de trabalho com séries parciais, cuja principal vantagem em relação à análise clássica de extremos, que amostra os máximos anuais para o ajuste da distribuição de Gumbel, é a amostragem de uma maior quantidade de eventos extremos, já que a metodologia de amostragem prevê a utilização de todos os dados de vento acima de um determinado limite de magnitude.

Desta forma, a necessidade descrita por Cook (1985) de utilização de séries de vento com no mínimo 10 anos de extensão para estimação confiável dos parâmetros da

\section{Estações Meteorológicas Selecionadas}

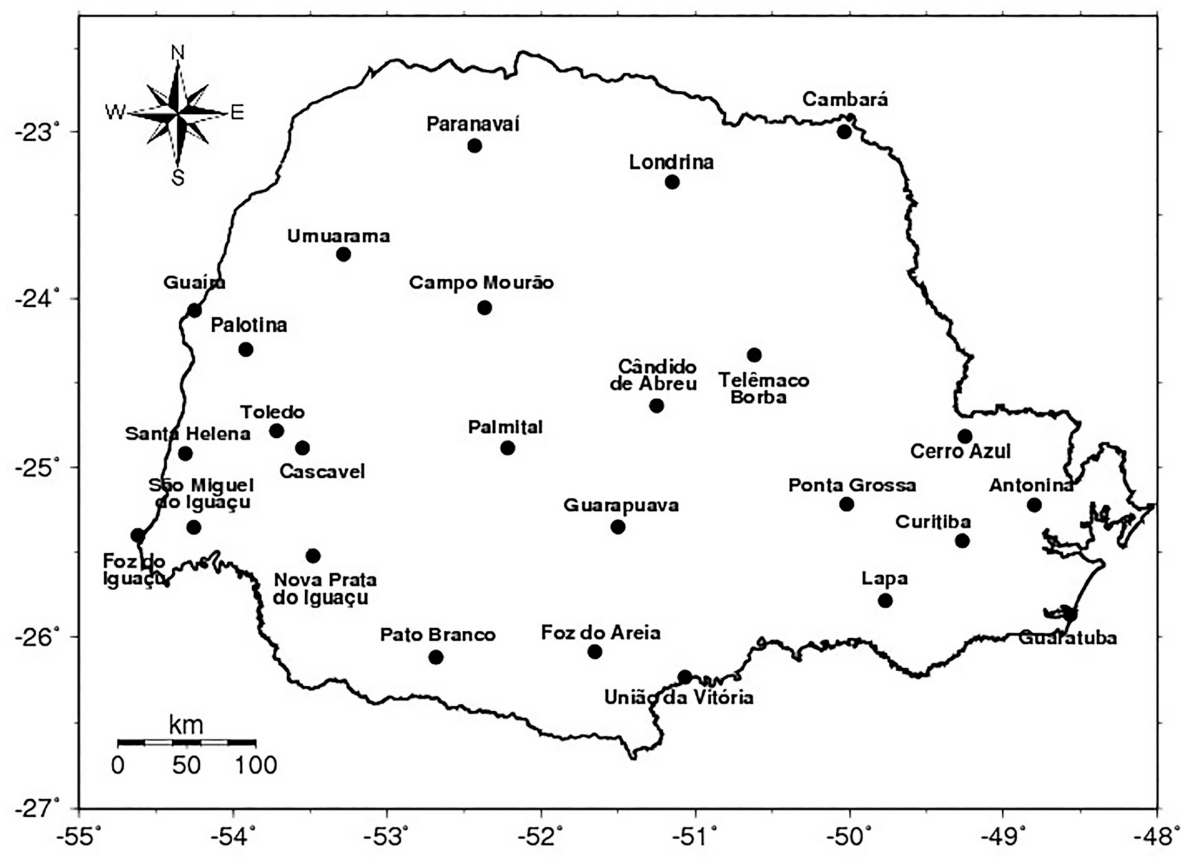

Figura 1 - Estações meteorológicas operadas pelo SIMEPAR e utilizadas neste estudo. 


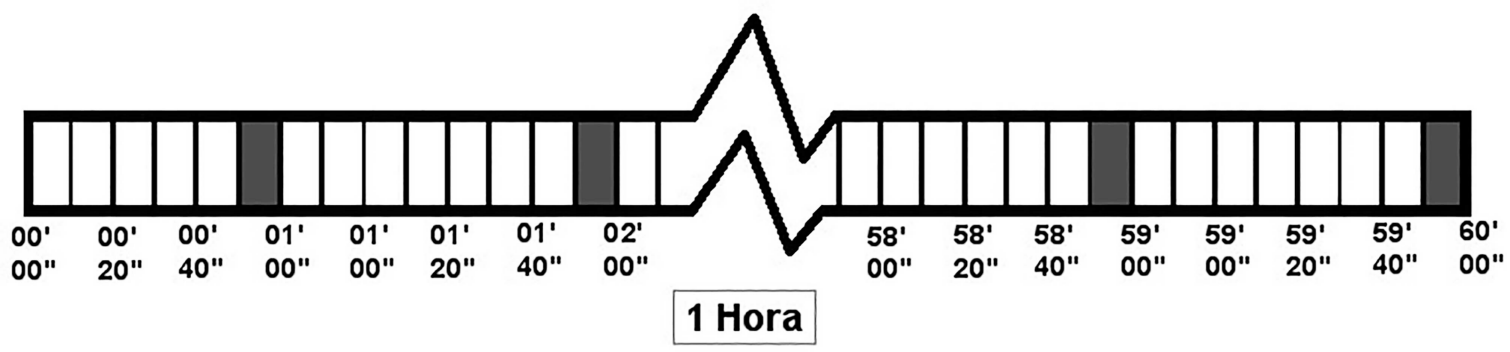

Média dos últimos 10" do minuto, utilizada para o cálculo da velocidade do vento

Figura 2 - Amostragem do dado de vento mais adequado para análise de extremos nas estações meteorológicas do SIMEPAR.

distribuição de extremos utilizando técnicas clássicas, como a amostragem do máximo vento anual e o ajuste da distribuição de extremos de Gumbel, pode ser atendida com séries de 5 a 6 anos (Palutikof et al., 1999) com a utilização de técnicas como modified independent storms (MIS) e peaks-over-threshold e o ajuste de distribuições de extremos próprias para análises de séries parciais.

De acordo com a estratégia de trabalho com séries parciais, foram selecionadas velocidades de ventos extremos por meio do método MIS descrito por Palutikof et al. (1999) e An e Pandey (2005). Na aplicação do método, foi adotado um intervalo mínimo de oito dias para a seleção de dois eventos consecutivos, visando garantir a independência das amostras. A Fig. 3 apresenta um exemplo de seleção das velocidades extremas de vento.
O método de análise de frequência regional aplicado neste estudo foi baseado nas proposições de Hosking e Wallis (1997). Segundo estes autores, a análise de frequência regional pode ser conduzida da seguinte forma: teste de homogeneidade regional; seleção da distribuição de frequência; e estimativa de distribuição.

Para identificar as estações meteorológicas cujos dados apresentam similaridades, constituindo-se regiões homogêneas na área de estudo, foi utilizada inicialmente a estatística descritiva, em seguida, o método de Análise dos Componentes Principais e, finalmente, o teste de homogeneidade regional, de acordo com a medida e critérios de heterogeneidade propostos por Hosking e Wallis (1997). Para implementação dessa última técnica, foi utilizada a rotina computacional produzida por Hosking (2005).

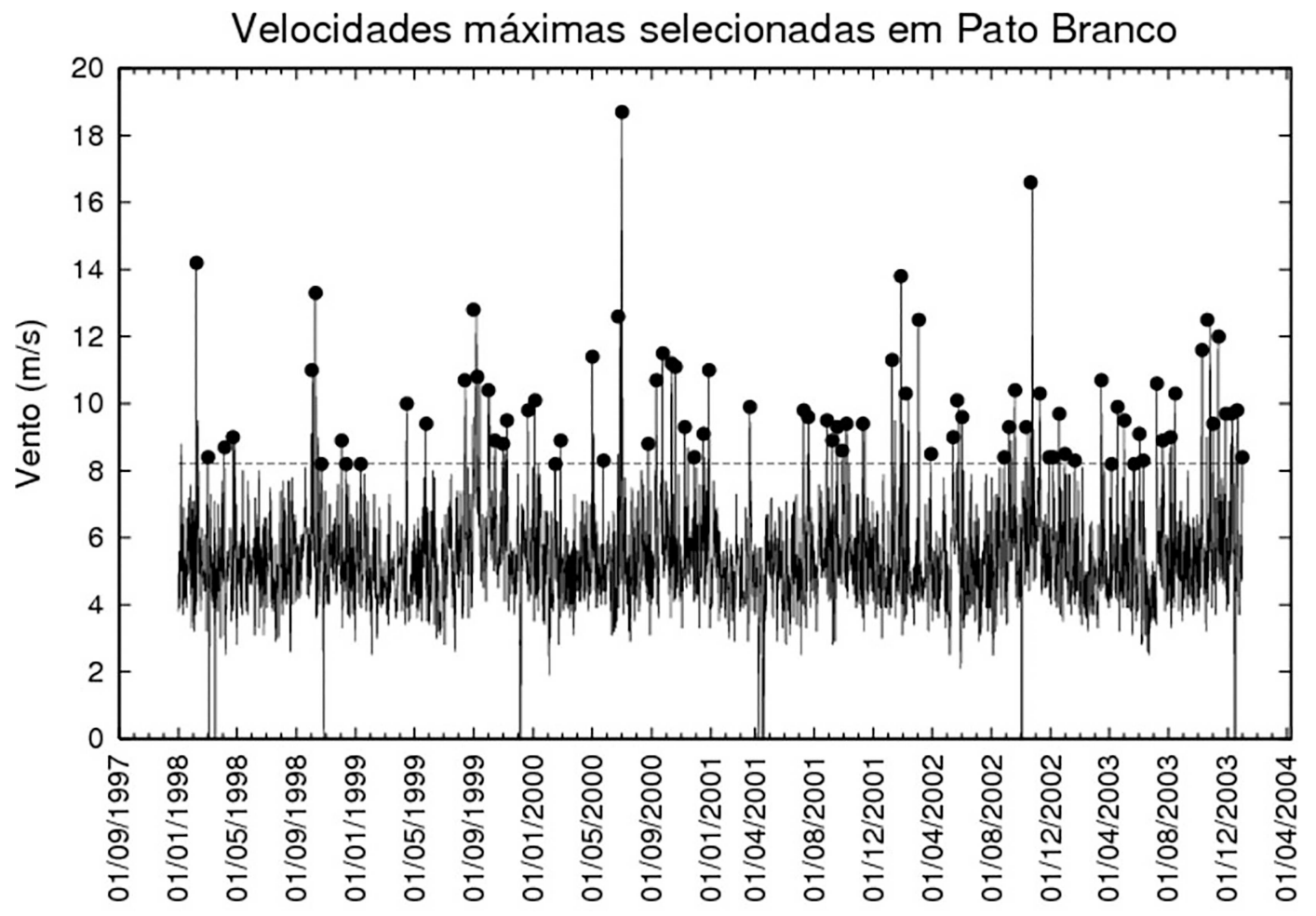

Figura 3 - Amostra de velocidades de vento (ćŕrculos pretos) selecionadas. 
O teste de homogeneidade regional foi realizado por meio da medida de discordância e da medida de heterogeneidade. No cálculo da discordância se supõe que há $N$

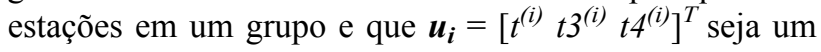
vetor que contém os valores de $t, t_{3}$ e $t_{4}$ (momentos- $L$ amostrais) de cada estação $\boldsymbol{i}$. Então, se calcula a média de $\boldsymbol{u}$ para cada região:

$$
\bar{u}=N^{-1} \sum_{i=1}^{N} u_{i}
$$

Define-se a matriz de soma dos quadrados e produtos cruzados:

$$
D_{i}=\frac{1}{3} N\left(u_{i}-\bar{u}\right)^{T} A^{-1}\left(u_{i}-\bar{u}\right)
$$

O critério inicialmente sugerido por Hosking e Wallis (1997) para definir se uma estações discorda das demais de sua região é $D_{i} \geq 3$. Mas em regiões com 10 estações ou menos, Hosking e Wallis (1997) propõem o seguinte critério:

$$
D_{i} \leq \frac{(N-1)}{3}
$$

No cálculo da medida de heterogeneidade, supôs-se que a região proposta tem $N$ estações, cujas séries de dados têm extensão $n_{i}$ e momentos-L estimados $t^{(i)}, t_{3}{ }^{(i)}$ e $t_{4}{ }^{(i)}$. Utilizou-se a Eq. (4), denotando-se por $t^{R}, t_{3}{ }^{R}$ e $t_{4}{ }^{R}$ as médias regionais de $L$-CV, L-skewness e L-kurtosis, ponderadas pela extensão da série de dados das estações.

$$
t^{R}=\frac{\sum_{i=1}^{N} n_{i} t^{(i)}}{\sum_{i=1}^{N} n_{i}}
$$

Calculou-se o desvio-padrão ponderado dos L-CVs amostrais de cada estação,

$$
V=\left\{\frac{\sum_{i=1}^{N} n_{i}\left(t^{(i)}-t^{R}\right)^{2}}{\sum_{i=1}^{N} n_{i}}\right\}^{1 / 2}
$$

Ajustou-se uma distribuição kappa aos momentos- $L$ regionais $t^{R}, t_{3}{ }^{R}$ e $t_{4}{ }^{R}$. Simulou-se um grande número de realizações $N_{\text {sim }}$ de uma região com $N$ estações, cada uma com a distribuição kappa ajustada como sendo sua distribuição de frequências. Para cada região simulada, calculou-se $V$ e determinou-se a média $\left(\mu_{V}\right)$ e o desvio-padrão $\left(\sigma_{V}\right)$ dos
$N_{\text {sim }}$ valores de $V$. Calculou-se a medida de heterogeneidade:

$$
H=\frac{\left(V-\mu_{V}\right)}{\sigma_{V}}
$$

Determinou-se as regiões homogêneas utilizando os seguintes critérios (Hosking e Wallis, 1997):

- Se $H<1$, a região é "aceitavelmente homogênea";

- Se $1 \leq H<2$, a região é "possivelmente heterogênea";

- Se $H \geq 2$, a região é "definitivamente heterogênea".

Foram utilizadas neste estudo as distribuições de extremos de Gumbel, Generalizada de Valores Extremos (GEV), Pareto generalizada, Logística generalizada, Lognormal, Pearson tipo III, Wakeby e Kappa) e o respectivo método de determinação dos momentos- $L$.

Para selecionar a distribuição de frequência visando a análise regional ajustou-se cada distribuição aos seus momentos-L regionais $\left(l, t^{R}\right.$ e $\left.t_{3}{ }^{R}\right)$. Denotou-se por $\tau_{4}{ }^{\text {DIST }}$ a L-kurtosis da distribuição ajustada, onde DIST é a abreviatura de uma distribuição, por exemplo GUM (Gumbel) e GEV. Ajustou-se uma distribuição kappa de quatro parâmetros às taxas médias regionais do momentos- $L l, t^{R}, t_{3}{ }^{R}$ e $t_{4}{ }^{R}$.

Foi simulado um elevado número, $N_{\text {sim }}$, de realizações de uma região com $N$ locais, cada qual com distribuição kappa como sua frequência regional. As regiões simuladas são homogêneas e não apresentam correlações cruzadas ou em série. As estações simuladas têm a mesma extensão de série que suas correspondentes reais. Para cada região $m$, calculou-se a média regional do L-skewness $t_{3}^{[m]}$, do L-kurtosis $t_{4}^{[m]}$ e o bias de $t_{4}{ }^{R}$,

$$
B_{4}=N_{\text {sim }}^{-1} \sum_{m=1}^{N_{s i m}}\left(t_{4}^{[m]}-t_{4}^{R}\right)
$$

o desvio-padrão de $t_{4}{ }^{R}$ foi calculado como

$$
\sigma_{4}=\left[\left(N_{\text {sim }}-1\right)^{-1}\left\{\sum_{m=1}^{N_{\text {sim }}}\left(t_{4}^{[m]}-t_{4}^{R}\right)^{2}-N_{\text {sim }} B_{4}^{2}\right\}\right]^{1 / 2}
$$

e, para cada distribuição, a medida de aderência do ajuste foi calculada como

$$
Z^{D I S T}=\frac{\left(\tau_{4}^{D I S T}-t_{4}^{R}+B_{4}\right)}{\sigma_{4}}
$$

O critério usado para definir se a distribuição testada apresentou um ajuste adequado foi adotar os resultados em que $\left|Z^{\mathrm{DIST}}\right| \leq 1,64$ (Hosking e Wallis, 1997).

Para a estimativa da distribuição foi realizada a estimativa dos momentos-L regionais, de acordo com a proposta de Hosking e Wallis (1997), utilizando-se as 
Eqs. (4)-(10):

$$
t_{r}^{R}=\frac{\sum_{i=1}^{N} n_{i} t_{r}^{(i)}}{\sum_{i=1}^{N} n_{i}}
$$

Determinou-se a média regional como sendo $l_{l}^{R}=1$. Ajustou-se a distribuição conforme apresentado anteriormente e denotou-se por $\hat{q}($.$) a função quantil da dis-$ tribuição de frequência regional ajustada. Os quantis na estação $i$ foram obtidos combinando-se as estimativas de $\mu_{i}$ e $q($.$) . A estimativa do quantil com probabilidade de$ não-excedência $F$ foi calculada como:

$$
\hat{Q}_{i}(F)=l_{1}^{(i)} \hat{q}(F)
$$

Para avaliar a acurácia dos quantis utilizou-se a metodologia de Hosking e Wallis (1997).

\section{Resultados}

As primeiras análises da série de ventos foram conduzidas com o uso de estatísticas descritivas e do ajuste de distribuições de probabilidade, às rajadas máximas anuais de estações, em regiões geográficas distintas no Estado do Paraná. A Fig. 4 ilustra as estações selecionadas.
Em busca do comportamento padrão das velocidades do vento em situações de rajada extrema (rajadas cujas velocidades superam $99 \%$ dos registros), foram produzidas composições que compreendem um período entre 5 horas antes da rajada extrema e 5 horas após este registro, conforme ilustrado na Fig. 5. Ao analisar o conjunto de composições das rajadas máximas de vento em todas as regiões definidas neste estudo percebe-se que, em geral, o pico de rajada (via de regra, de 20 a $25 \mathrm{~m} \cdot \mathrm{s}^{-1}$ ) é precedido e sucedido por baixas velocidades de rajada de vento (em torno de $10 \mathrm{~m} \cdot \mathrm{s}^{-1}$ ). Entretanto, há estações em que isto não ocorre na totalidade dos eventos extremos, seja por diferenças nas condições geográficas ou nos sistemas meteorológicos atuantes.

A análise de extremos foi realizada utilizando todas as distribuições de extremos descritas em material e métodos. Entretanto, os resultados apresentados correspondem aos ajustes das distribuições Gumbel (2 parâmetros), GEV (3 parâmetros) e Wakeby (5 parâmetros). Esta seleção foi escolhida por apresentar comportamentos de distribuições em ordem crescente de complexidade e, por conseguinte, ordem crescente de incerteza em seus ajustes (especialmente ao considerar que a série de rajadas usada neste estudo é de apenas 8 anos). Além disso, as distribuições Gumbel e GEV são comumente usadas em análises de extremos, haja vista a norma brasileira de projetos de linhas aéreas de transmissão de energia elétrica (ABNT, 1985).

As estações selecionadas para apresentação das distribuições de extremos são as mesmas da Fig. 4. Isto

\section{Estações Meteorológicas Selecionadas}

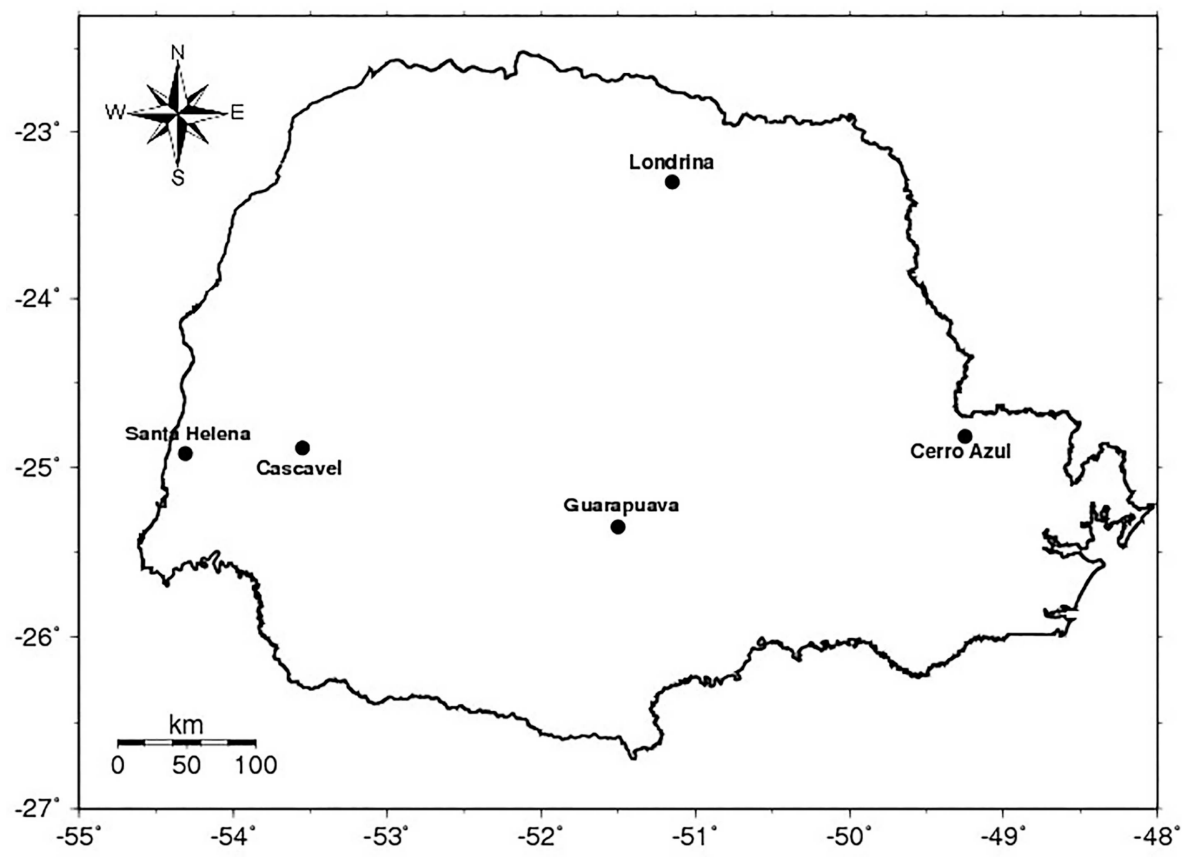

Figura 4 - Estações meteorológicas selecionadas para a estatística descritiva. 
Composição das Rajadas Máximas Anuais - Cerro Azul

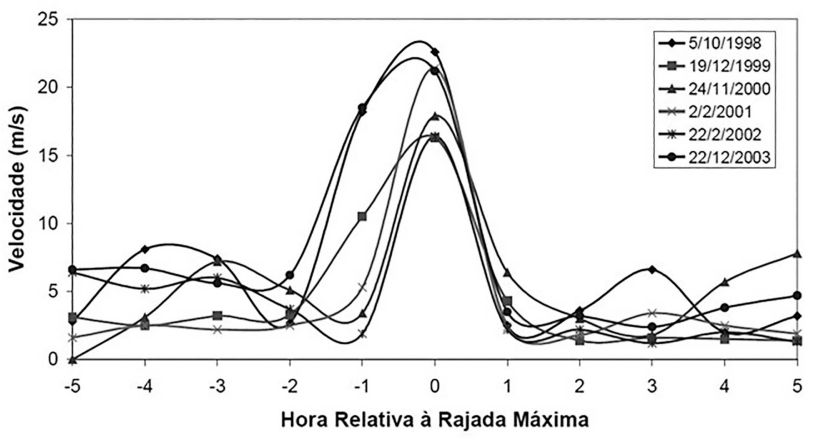

Composição das Rajadas Máximas Anuais - Guarapuava

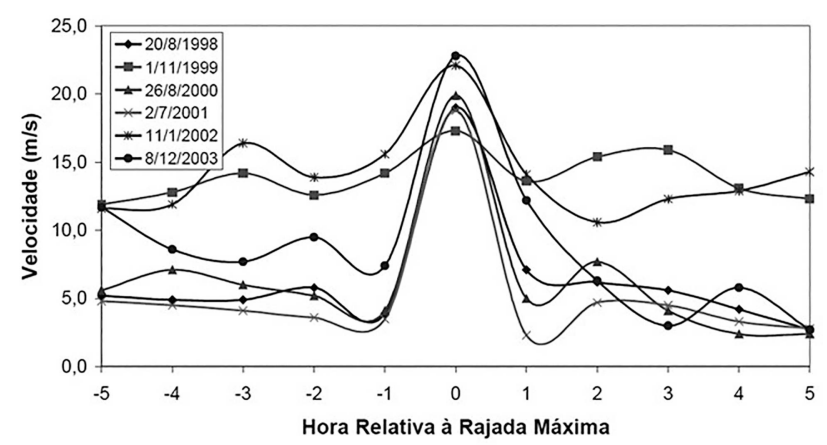

Composição das Rajadas Máximas Anuais - Londrina

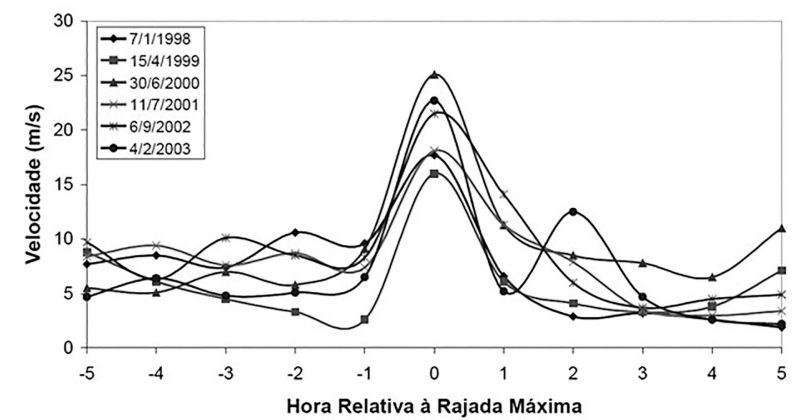

Composição das Rajadas Máximas Anuais - Cascavel

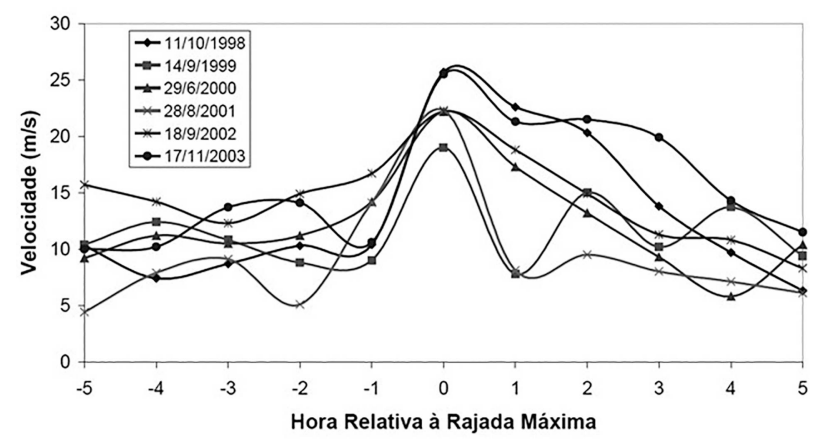

Figura 5 - Composição das rajadas máximas anuais nas estações selecionadas.
Composição das Rajadas Máximas Anuais - Santa Helena

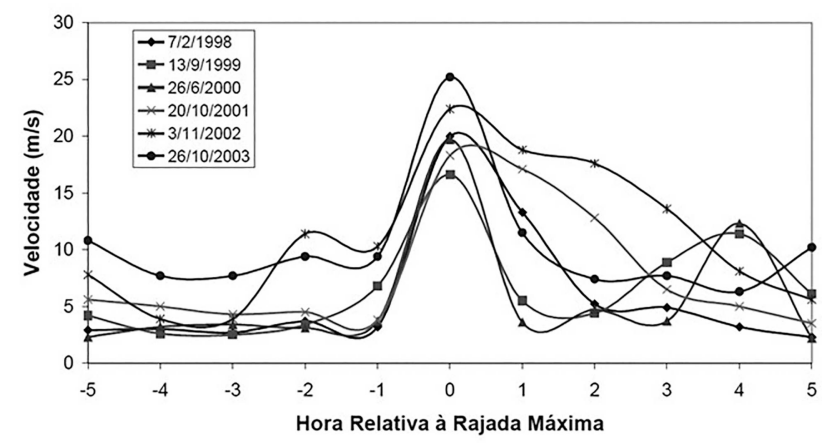

porque o propósito é suportar o diagnóstico da necessidade de análise regional, ou não, da frequência de ventos extremos no Paraná.

O ajuste da distribuição de Gumbel aos dados das cinco estações selecionadas enfatizou as diferenças nas características dos dados. Na Fig. 6 (esq.) percebe-se diferença significativa na curtose da distribuição ajustada para estações como Londrina e Cascavel (cuja distância entre elas é de $390 \mathrm{~km}$ aproximadamente).

Na Fig. 6 (dir.), que ilustra as funções distribuição acumulada de Gumbel ajustadas para as cinco estações, são perceptíveis diferenças em todo o intervalo de probabilidade (0 a 1). Além disso, as diferenças mais significativas encontram-se na estimativa dos quantis extremos, foco deste estudo. Um exemplo desta ocorrência é verificado ao comparar os quantis estimados com probabilidade de não-excedência de 0,98 , em Guarapuava e Londrina. Enquanto na primeira estação o quantil obtido foi de aproximadamente $31 \mathrm{~m} \cdot \mathrm{s}^{-1}$, na segunda foi próximo a $39 \mathrm{~m} \cdot \mathrm{s}^{-1}$.

O ajuste da distribuição GEV aos dados das cinco estações selecionadas produziu quantis ainda mais distintos a que os produzidos pela distribuição de Gumbel. Na Fig. 7 (dir.) percebe-se que as caudas da direita das funções GEV ajustadas aos máximos anuais das estações selecionadas não apresentam o mesmo comportamento. $\mathrm{O}$ que indica a existência de uma diferença significativa nas características dos ventos extremos entre estas estações.

Na Fig. 7 (esq.), que ilustra as funções distribuição acumulada GEV ajustadas para as cinco estações, são perceptíveis diferenças em todo o intervalo de probabilidade (0 a 1). Além disso, as estimativas dos quantis para uma 
Distribuição de Extremos Gumbel Ajustada por Estação

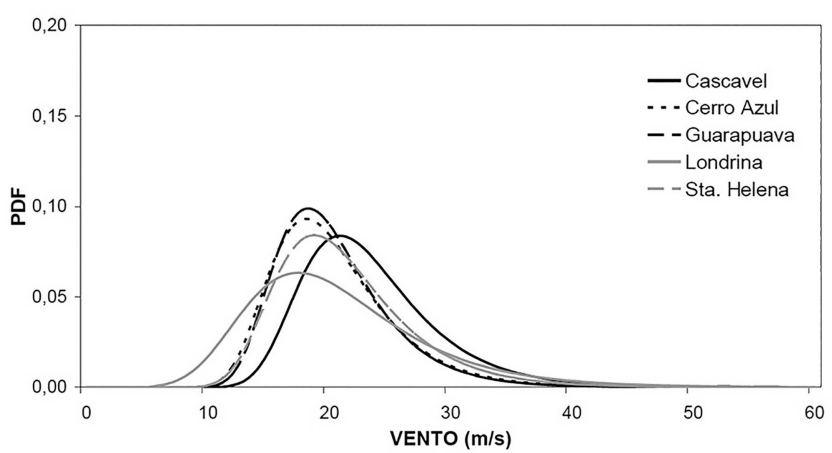

Distribuição Gumbel Acumulada Ajustada por Estação

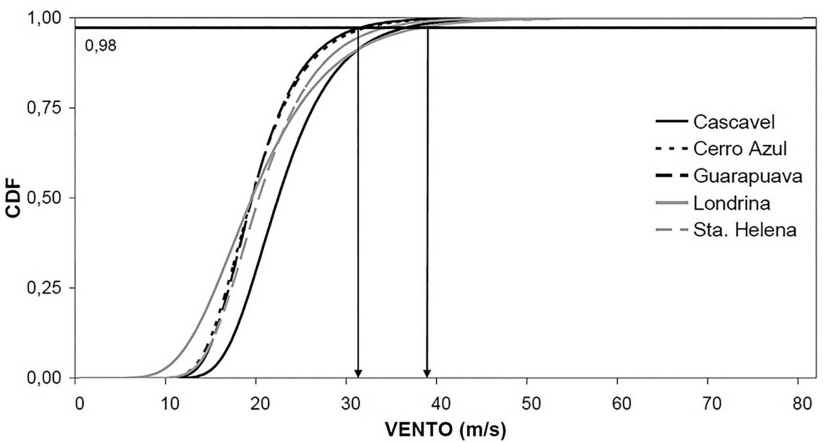

Figura 6 - Funções distribuição de probabilidade (à esquerda) e distribuição acumulada (à direita) de Gumbel ajustadas aos máximos anuais das estações: Cascavel, Cerro Azul, Guarapuava, Londrina e Santa Helena.
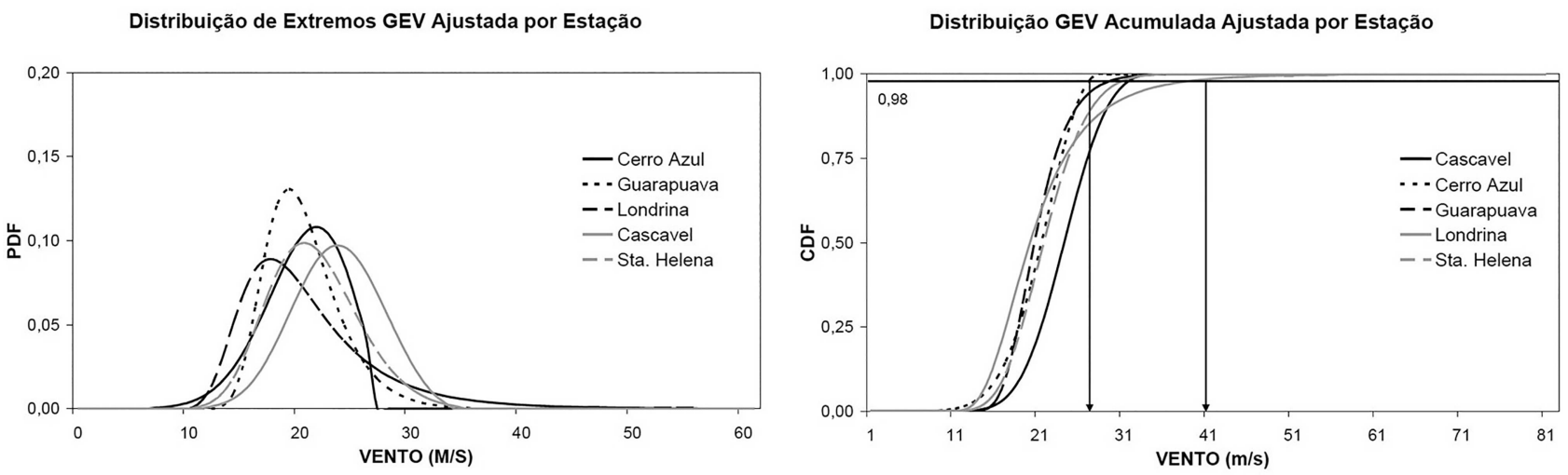

Figura 7 - Funções distribuição de probabilidade (à esquerda) e distribuição acumulada (à direita) GEV ajustadas aos máximos anuais das estações: Cascavel, Cerro Azul, Guarapuava, Londrina e Santa Helena.

probabilidade de não-excedência de 0,98 apresentaram diferenças da ordem de $14 \mathrm{~m} . \mathrm{s}^{-1}$ entre o menor $\left(27 \mathrm{~m} . \mathrm{s}^{-1}\right.$ em Cerro Azul) e o maior (41 m.s ${ }^{-1}$ em Londrina) quantil estimado.

O ajuste da distribuição de Wakeby não-regionalizada aos dados das cinco estações selecionadas enfatizou

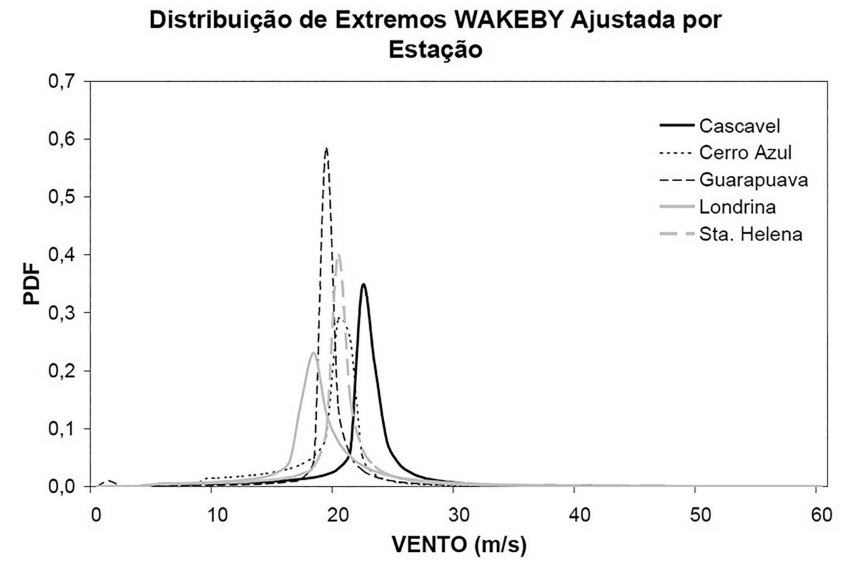

ainda mais as diferenças nas características dos dados. $\mathrm{Na}$ Fig. 8 percebe-se diferenças significativas em diversos momentos das distribuições, como média e desviopadrão. Nos quantis estimados com probabilidade de nãoexcedência de 0,98 , obteve-se $27 \mathrm{~m} \cdot \mathrm{s}^{-1}$ em Cerro Azul e $43 \mathrm{~m} . \mathrm{s}^{-1}$ em Londrina.

Distribuição Wakeby Acumulada Ajustada por Estação

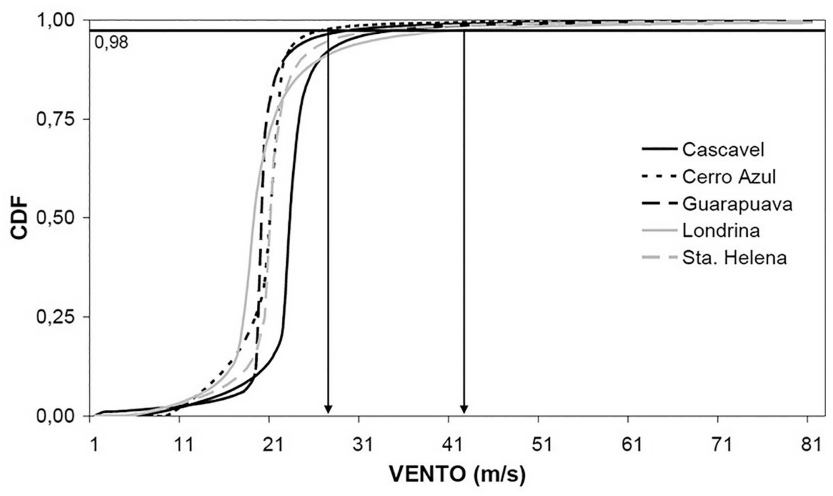

Figura 8 - Funções distribuição de probabilidade (à esquerda) e distribuição acumulada (à direita) Wakeby ajustadas aos máximos anuais das estações: Cascavel, Cerro Azul, Guarapuava, Londrina e Santa Helena. 
Ao analisar comparativamente os resultados obtidos com o ajuste da distribuição de Wakeby e os resultados obtidos com as distribuições de Gumbel e GEV, percebese que a medida em que a complexidade da distribuição aumenta (2, 3 e 5 parâmetros), as diferenças verificadas (1) nas estimativa dos quantis e (2) nas características das funções de distribuição de probabilidade tornam-se maiores.

Para investigar a suspeita de significativas diferenças nas características dos dados de estações em regiões geográficas distintas, foram realizados a análise de componentes principais e o teste de homogeneidade regional para as 26 estações meteorológicas consideradas no estudo. A identificação inicial das regiões homogêneas foi fundamentada na homogeneidade obtida na estatística descritiva.

A Fig. 9 apresenta as porcentagens de variância aplicada de acordo com o número de componentes principais. Neste estudo foram considerados sete componentes principais, que explicam $65 \%$ da variância total.

A partir dos carregamentos obtidos e apresentados na Tabela 2, foi possível diagnosticar estações cujas rajadas apresentam padrões de similaridade entre si. Para cada componente principal foram destacadas as estações que produziram os maiores carregamentos.

\section{Variância Acumulada Explicada pelos CPs}

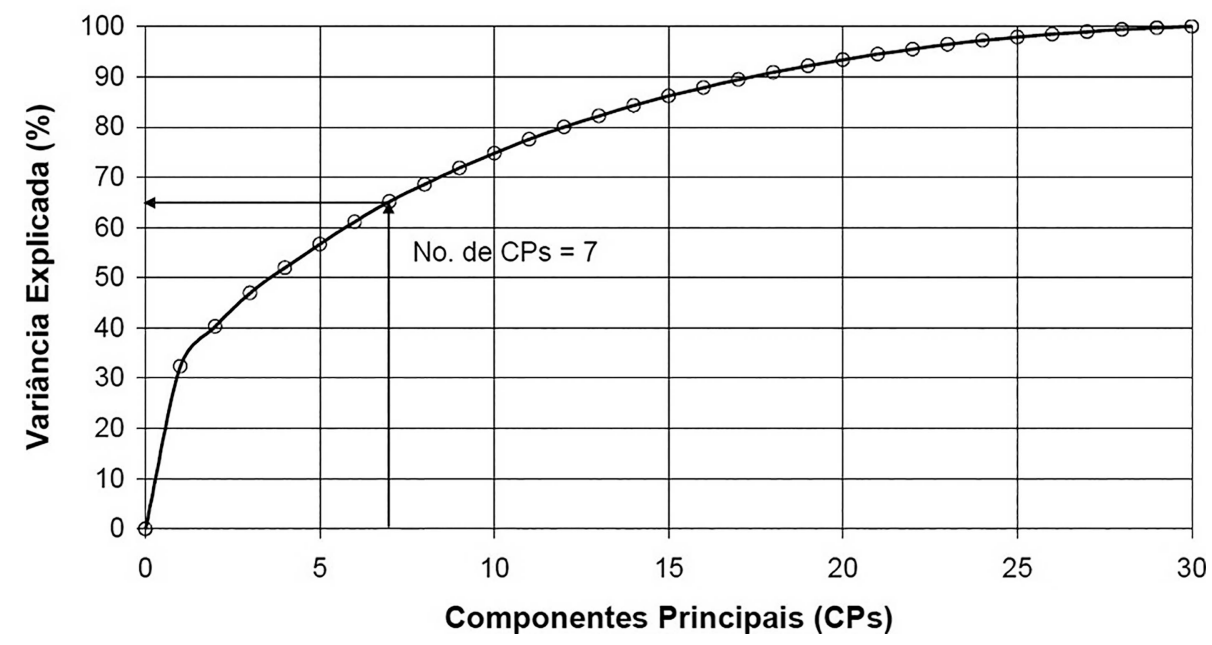

Figura 9 - Porcentagem da variância explicada em função do número de componentes principais.

Tabela 2 - Resultados dos carregamentos.

\begin{tabular}{|c|c|c|c|c|c|c|c|}
\hline Estação & $\mathrm{CP}-1$ & $\mathrm{CP}-2$ & $\mathrm{CP}-3$ & $\mathrm{CP}-4$ & $\mathrm{CP}-5$ & $\mathrm{CP}-6$ & $\mathrm{CP}-7$ \\
\hline Antonina & 0,12 & 0,04 & $-0,02$ & 0,27 & 0,49 & 0,23 & $-0,07$ \\
\hline Cândido de Abreu & 0,33 & 0,02 & $\mathbf{0 , 3 3}$ & 0,06 & 0,35 & $-0,02$ & 0,46 \\
\hline Cambará & $-0,01$ & 0,10 & $\mathbf{0 , 7 7}$ & 0,15 & 0,17 & 0,09 & 0,01 \\
\hline Cascavel & 0,27 & 0,43 & 0,29 & $\mathbf{0 , 3 0}$ & 0,06 & 0,01 & 0,04 \\
\hline Cerro Azul & 0,19 & 0,11 & 0,14 & 0,08 & $\mathbf{0 , 5 3}$ & 0,11 & 0,19 \\
\hline Campo Mourão & $\mathbf{0 , 3 7}$ & 0,15 & 0,32 & 0,09 & 0,26 & 0,09 & 0,02 \\
\hline Curitiba & $\mathbf{0 , 3 1}$ & $-0,03$ & 0,19 & 0,23 & 0,21 & 0,23 & 0,20 \\
\hline Foz do Areia & $\mathbf{0 , 3 3}$ & 0,16 & 0,00 & 0,08 & 0,10 & 0,03 & 0,92 \\
\hline Foz do Iguaçu & $-0,11$ & $\mathbf{0 , 3 9}$ & 0,25 & 0,36 & 0,27 & 0,03 & 0,31 \\
\hline Guaíra & 0,15 & 0,40 & 0,37 & 0,05 & 0,29 & 0,23 & 0,20 \\
\hline Guarapuava & 0,60 & 0,13 & 0,08 & 0,12 & 0,06 & 0,16 & 0,21 \\
\hline Guaratuba & $-0,02$ & 0,11 & 0,09 & $-0,01$ & 0,65 & 0,05 & 0,05 \\
\hline Londrina & 0,19 & 0,11 & $\mathbf{0 , 3 4}$ & 0,13 & 0,36 & $\mathbf{0 , 8 3}$ & 0,00 \\
\hline Nova Prata do Iguaçu & 0,45 & 0,33 & 0,05 & 0,34 & 0,08 & 0,19 & 0,17 \\
\hline Palmas & $\mathbf{0 , 4 3}$ & 0,26 & 0,12 & 0,46 & $-0,04$ & 0,23 & 0,27 \\
\hline
\end{tabular}


Tabela 2 - continua

\begin{tabular}{|c|c|c|c|c|c|c|c|}
\hline Estação & CP-1 & CP-2 & $\mathrm{CP}-3$ & CP-4 & CP-5 & CP-6 & $\mathrm{CP}-7$ \\
\hline Palmital & 0,66 & 0,12 & 0,11 & 0,07 & 0,03 & $-0,04$ & 0,08 \\
\hline Palotina & 0,05 & 0,61 & 0,12 & 0,35 & 0,01 & 0,21 & 0,14 \\
\hline Paranavaí & 0,15 & 0,34 & 0,57 & 0,10 & $-0,01$ & 0,12 & 0,10 \\
\hline Pato Branco & 0,51 & 0,22 & 0,02 & 0,27 & 0,12 & 0,21 & 0,23 \\
\hline Ponta Grossa & 0,38 & 0,30 & 0,25 & 0,15 & 0,32 & $-0,20$ & 0,01 \\
\hline Santa Helena & 0,27 & 0,94 & 0,02 & 0,10 & 0,15 & $-0,02$ & 0,00 \\
\hline São Miguel do Iguaçu & 0,18 & 0,54 & 0,33 & 0,12 & 0,02 & $-0,04$ & 0,08 \\
\hline Telêmaco Borba & 0,28 & 0,14 & 0,40 & 0,02 & 0,17 & 0,38 & 0,09 \\
\hline Toledo & 0,13 & 0,23 & 0,22 & 0,81 & 0,15 & 0,00 & 0,03 \\
\hline Umuarama & 0,50 & 0,06 & 0,50 & 0,11 & $-0,01$ & 0,16 & 0,03 \\
\hline União da Vitória & $\mathbf{0 , 3 3}$ & $-0,09$ & 0,01 & 0,13 & 0,35 & 0,35 & 0,14 \\
\hline
\end{tabular}

Analisando-se os resultados na Tabela 2 e as regiões delimitadas na Fig. 10 percebe-se que o primeiro componente principal (CP-1) é o que engloba a maior quantidade de estações e o responsável pela maior parcela de variância explicada $(32,4 \%)$. Na sequência, os componentes principais englobam cada vez menos estações e explicam parcelas decrescentes da variância total.

Para a produção dos testes de homogeneidade regional foi utilizada a rotina computacional produzida por Hosking (2005) e chamada de xtest.

Em seguida, calculou-se a medida de discordância, com os resultados apresentados na Tabela 3. Percebe-se que as estações que apresentaram discordância com suas respectivas regiões, encontram-se em áreas com poucas estações (3 ou menos), com exceção de São Miguel do Iguaçu. Tais resultados mostram que o teste tem aplicabilidade em regiões com mais de três estações, tornando-se vulnerável em regiões com poucas estações.

Em complemento às medidas de discordância, calculou-se as medidas de heterogeneidade. Os resultados apresentados na Tabela 4 mostram que todas as regiões atendem o critério proposto da Eq. (6), com exceção das medidas obtidas em $\boldsymbol{H}_{3}$. Tais heterogeneidades em $H_{3}$ foram obtidas nas regiões mais extensas definidas pela

\section{Identificação de Regiões Homogêneas - Método ACP}

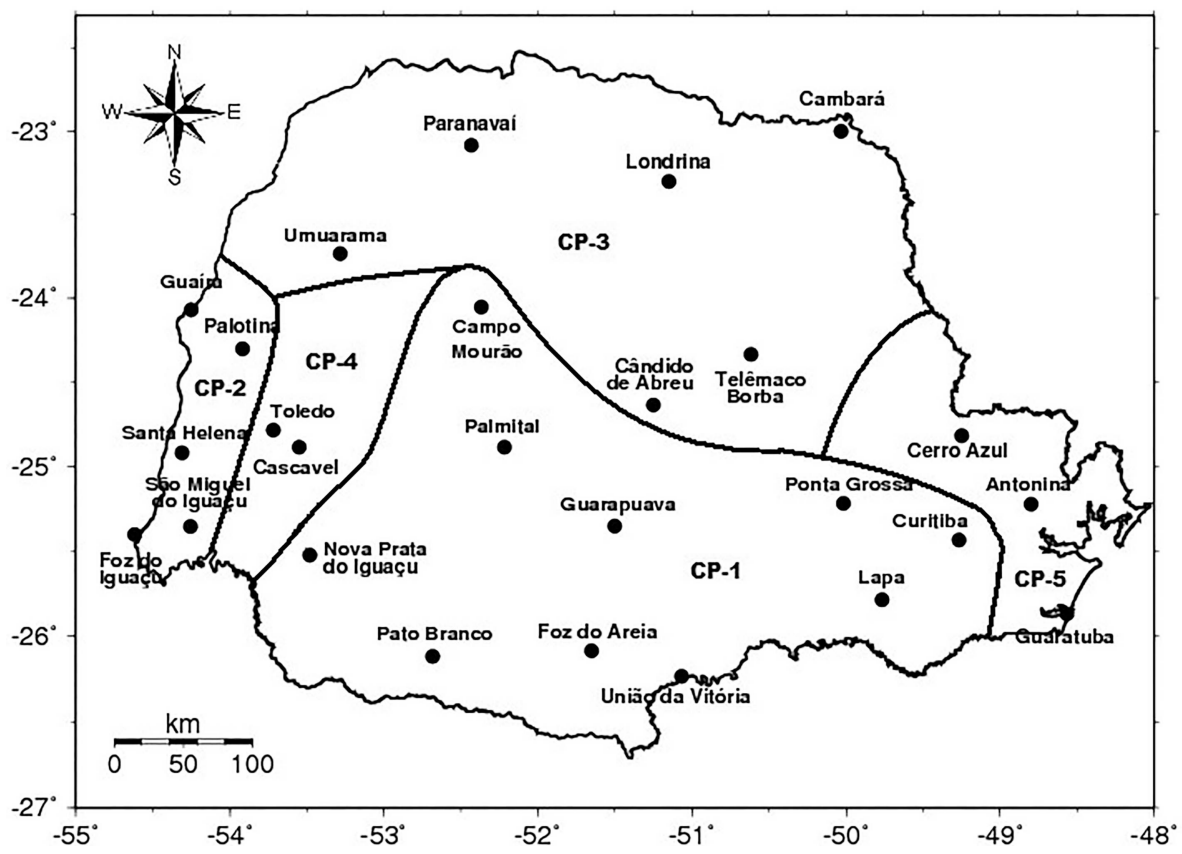

Figura 10 - Regiões paranaenses cujos padrões de rajadas foram indicados como semelhantes, de acordo com a análise de componentes principais. 
Tabela 3 - Resultados do teste de discordância.

\begin{tabular}{|c|c|c|c|c|c|}
\hline Região & Estação & $\mathrm{L}-\mathrm{CV}$ & L-SKEW & L-KURT & $\mathrm{D}(\mathrm{I})$ \\
\hline & TODAS & 2,9988 & 0,3886 & 0,6596 & - \\
\hline \multirow[t]{11}{*}{ CP-1 } & Campo Mourão & 3,4820 & 0,4444 & 0,5234 & 1,73 \\
\hline & Curitiba & 2,1156 & 0,4329 & 0,7849 & 0,95 \\
\hline & Foz do Areia & 2,4065 & 0,3905 & 0,7197 & 0,28 \\
\hline & Guarapuava & 2,5718 & 0,4484 & 0,7393 & 0,54 \\
\hline & Lapa & 2,7488 & 0,4749 & 0,7538 & 0,85 \\
\hline & Nova Prata do Iguaçu & 3,2956 & 0,2749 & 0,5526 & 1,32 \\
\hline & Palmital & 3,0422 & 0,3982 & 0,6642 & 0,01 \\
\hline & Pato Branco & 2,6452 & 0,4096 & 0,7639 & 0,58 \\
\hline & Ponta Grossa & 2,9950 & 0,3379 & 0,5980 & 0,40 \\
\hline & União da Vitória & 2,7757 & 0,4715 & 0,6872 & 0,73 \\
\hline & Média Ponderada CP-1 & 2,8078 & 0,4083 & 0,6787 & máx.: 3,00 \\
\hline \multirow[t]{6}{*}{$\mathrm{CP}-2$} & Foz do Iguaçu & 2,8559 & 0,4346 & 0,7112 & 0,25 \\
\hline & Guaíra & 2,6949 & 0,383 & 0,6836 & 0,07 \\
\hline & Palotina & 3,4318 & 0,3691 & 0,6537 & 0,44 \\
\hline & Santa Helena & 3,0292 & 0,3618 & 0,6386 & 0,07 \\
\hline & São Miguel do Iguaçu & 4,8748 & 0,4154 & 0,5237 & 2,80 \\
\hline & Média Ponderada CP-2 & 3,3773 & 0,3928 & 0,6422 & máx.: 1,33 \\
\hline \multirow[t]{7}{*}{$\mathrm{CP}-3$} & Cambará & 3,1309 & 0,3702 & 0,6449 & 0,05 \\
\hline & Cândido de Abreu & 2,8109 & 0,4741 & 0,7309 & 0,69 \\
\hline & Londrina & 4,0252 & 0,4715 & 0,5529 & 1,22 \\
\hline & Paranavaí & 1,8121 & 0,3095 & 0,7770 & 1,35 \\
\hline & Telêmaco Borba & 2,7526 & 0,3927 & 0,6585 & 0,13 \\
\hline & Umuarama & 2,9016 & 0,2574 & 0,5995 & 1,33 \\
\hline & Média Ponderada CP-3 & 2,9056 & 0,3792 & 0,6606 & máx.: 1,67 \\
\hline \multirow[t]{3}{*}{$\mathrm{CP}-4$} & Cascavel & 3,0388 & 0,3233 & 0,6323 & 0,34 \\
\hline & Toledo & 4,6885 & 0,4646 & 0,5917 & 2,74 \\
\hline & Média Ponderada CP4 & 3,8637 & 0,394 & 0,6120 & máx.: $\mathbf{0 , 3 3}$ \\
\hline \multirow[t]{4}{*}{$\mathrm{CP}-5$} & Antonina & 2,6106 & 0,4301 & 0,5772 & 2,45 \\
\hline & Cerro Azul & 2,7338 & 0,2475 & 0,5683 & 1,89 \\
\hline & Guaratuba & 2,3722 & 0,3125 & 0,8304 & 2,80 \\
\hline & Média Ponderada CP-5 & 2,5795 & 0,3263 & 0,6545 & máx.: 0,67 \\
\hline
\end{tabular}

Tabela 4 - Resultados das medidas de heterogeneidade.

\begin{tabular}{lccc}
\hline Região & $H_{1}$ & $H_{2}$ & $H_{3}$ \\
\hline CP-1 & $-0,26$ & $-0,27$ & $-2,57$ \\
CP-2 & $-0,22$ & $-0,22$ & $-2,07$ \\
CP-3 & $-0,20$ & $-0,21$ & $-1,91$ \\
CP-4 & $-0,13$ & $-0,13$ & $-0,83$ \\
CP-5 & $-0,21$ & $-0,21$ & $-0,50$ \\
\hline
\end{tabular}


análise de componentes principais, o que poderia sugerir, ainda que levemente, sub-divisões. Entretanto, para a manutenção da contiguidade geográfica optou-se pela manutenção das regiões.

Para a seleção da distribuição de frequência para análise regional, calculou-se a estatística de aderência do ajuste $Z^{\text {DIST }}$ para cada uma das 5 regiões homogêneas utilizando-se as seguintes distribuições de frequência: logística generalizada, GEV, normal generalizada, Pearson tipo III, Pareto generalizada e Wakeby. Com exceção da distribuição de Wakeby, todas as demais foram reprovadas no teste de seleção, ou seja, não atenderam o critério $\left|Z^{\mathrm{DIST}}\right| \leq 1,64$. Esta conclusão já tinha sido obtida no exemplo apresentado por Hosking e Wallis (1997) para os dados de vento dos EUA. Os quantis regionais estimados com o uso da distribuição de Wakeby estão apresentados na Tabela 5.

Para uma visualização espacial das diferentes magnitudes obtidas nos quantis das regiões CP-1 a CP-5 produziu-se a Fig. 11, com os resultados interpolados do quantil 0,98 obtido com a distribuição de Wakeby regionalizada em todas as estações.

Tabela 5 - Quantis estimados pela distribuição de Wakeby regionalizada e não regionalizada.

\begin{tabular}{|c|c|c|c|c|c|c|c|c|}
\hline \multirow[t]{2}{*}{ Estação } & \multicolumn{4}{|c|}{$\begin{array}{l}\text { Quantis obtidos com a distribuição de Wakeby não } \\
\text { regionalizada }\end{array}$} & \multicolumn{4}{|c|}{ Quantis obtidos com a distribuição de Wakeby regionalizada } \\
\hline & 0,90 & 0,95 & 0,98 & 0,99 & 0,90 & 0,95 & 0,98 & 0,99 \\
\hline \multicolumn{9}{|c|}{ Região CP-1 } \\
\hline Campo Mourão & 25,3 & 30,9 & 43,1 & 58,0 & 22,3 & 25,4 & 33,2 & 44,3 \\
\hline Curitiba & 19,4 & 21,3 & 26,3 & 33,7 & 20,3 & 23,1 & 30,1 & 40,1 \\
\hline Foz do Areia & 22,1 & 24,3 & 30,1 & 38,6 & 22,8 & 25,9 & 33,9 & 45,1 \\
\hline Guarapuava & 21,2 & 24,0 & 31,1 & 41,3 & 21,6 & 24,6 & 32,1 & 42,7 \\
\hline Lapa & 20,8 & 23,3 & 29,9 & 39,6 & 21,6 & 24,6 & 32,2 & 42,9 \\
\hline Nova Prata do Iguaçu & 26,6 & 30,0 & 38,4 & 49,9 & 25,7 & 29,3 & 38,2 & 50,9 \\
\hline Palmital & 22,9 & 26,0 & 33,9 & 45,3 & 23,0 & 26,1 & 34,2 & 45,5 \\
\hline Pato Branco & 20,4 & 22,1 & 26,6 & 33,6 & 21,7 & 24,7 & 32,3 & 43,0 \\
\hline Ponta Grossa & 23,6 & 27,1 & 35,7 & 47,2 & 22,8 & 25,9 & 33,9 & 45,1 \\
\hline União da Vitória & 19,6 & 22,9 & 31,2 & 42,9 & 19,4 & 22,1 & 28,8 & 38,4 \\
\hline \multicolumn{9}{|c|}{ Região CP-2 } \\
\hline Foz do Iguaçu & 21,9 & 24,8 & 32,3 & 43,0 & 22,8 & 26,4 & 35,3 & 47,8 \\
\hline Guaíra & 23,2 & 26,0 & 33,1 & 43,3 & 23,9 & 27,7 & 37,1 & 50,2 \\
\hline Palotina & 23,1 & 25,7 & 32,7 & 43,1 & 24,0 & 27,8 & 37,3 & 50,4 \\
\hline Santa Helena & 23,0 & 26,5 & 35,0 & 46,7 & 23,0 & 26,7 & 35,7 & 48,3 \\
\hline São Miguel do Iguaçu & 29,1 & 36,4 & 52,7 & 73,1 & 25,6 & 29,7 & 39,7 & 53,7 \\
\hline \multicolumn{9}{|c|}{ Região CP-3 } \\
\hline Cândido de Abreu & 21,6 & 24,5 & 32,1 & 43,1 & 22,2 & 25,3 & 33,2 & 44,3 \\
\hline Cambará & 24,6 & 28,0 & 36,5 & 48,4 & 24,5 & 28,0 & 36,6 & 48,9 \\
\hline Londrina & 25,1 & 31,4 & 45,6 & 63,5 & 22,2 & 25,3 & 33,2 & 44,2 \\
\hline Paranavaí & 16,3 & 17,1 & 19,3 & 22,8 & 17,6 & 20,1 & 26,4 & 35,2 \\
\hline Telêmaco Borba & 23,1 & 26,2 & 34,0 & 44,9 & 23,1 & 26,4 & 34,6 & 46,1 \\
\hline Umuarama & 21,8 & 24,4 & 31,1 & 40,5 & 21,6 & 24,6 & 32,3 & 43,1 \\
\hline \multicolumn{9}{|c|}{ Região CP-4 } \\
\hline Cascavel & 25,1 & 28,4 & 36,5 & 47,7 & 26,1 & 30,7 & 41,9 & 57,1 \\
\hline Toledo & 29,0 & 35,7 & 51,4 & 72,1 & 27,9 & 32,9 & 44,8 & 61,0 \\
\hline \multicolumn{9}{|c|}{ Região CP-5 } \\
\hline Antonina & 16,8 & 20,6 & 29,5 & 40,8 & 15,0 & 16,7 & 21,4 & 28,1 \\
\hline Cerro Azul & 21,3 & 22,8 & 26,9 & 33,2 & 21,5 & 24,0 & 30,6 & 40,3 \\
\hline Guaratuba & 19,7 & 20,3 & 22,0 & 24,7 & 21,4 & 24,0 & 30,6 & 40,2 \\
\hline
\end{tabular}




\section{Distribuição de Wakeby Regionalizada Quantil 0,98}

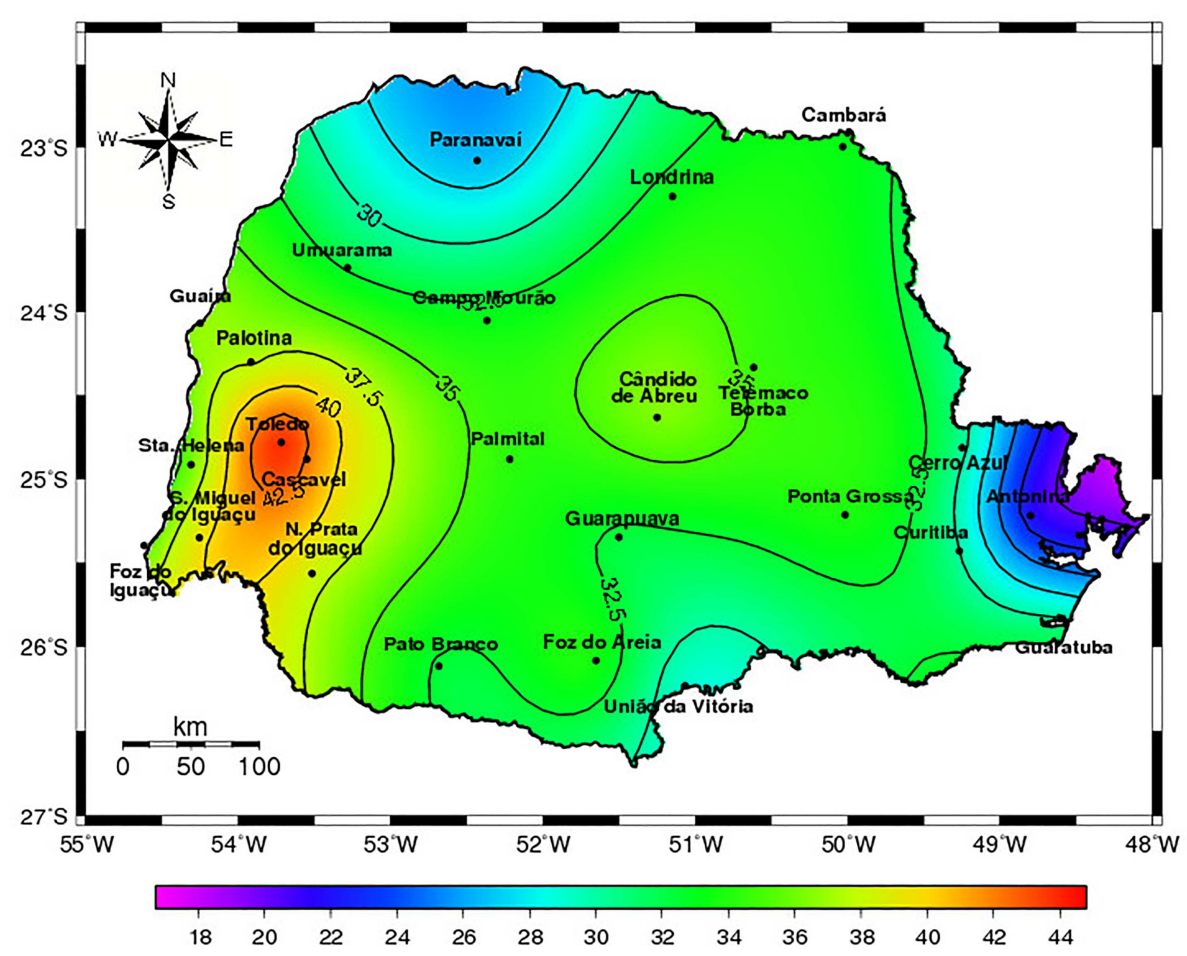

Figura 11 - Espacialização do quantil regional 0,98, correspondente a ventos máximos diários $\left(\mathrm{m} \cdot \mathrm{s}^{-1}\right)$ com tempo de recorrência de 50 anos.

\section{Conclusões}

A análise de frequência regional no Paraná utilizou séries curtas de rajadas ( 8 anos) em 26 estações ao longo do Estado para produzir conhecimento acerca da sazonalidade e autocorrelação diária dos ventos extremos no Estado; das regiões paranaenses com padrões de rajada homogêneos (litoral, central, meio-oeste, oeste e norte); e das distribuições de extremos mais adequadas para análise de frequência regional.

Foram identificadas cinco regiões homogêneas no Estado do Paraná, com resultados validados pelos testes de discrepância e de heterogeneidade descritos em Hosking e Wallis (1997), sendo geograficamente coerentes com as características de relevo do território paranaense.

Os testes de seleção das distribuições regionais, nos quais foram testadas distribuições de dois a cinco parâmetros, indicaram que apenas uma distribuição com menos graus de liberdade como a distribuição de Wakeby (cinco parâmetros) seria capaz de estimar quantis com razoável precisão. Os quantis regionais estimados exibiram elevada coerência com as diferenças espaciais diagnosticadas na estatística descritiva. Esta coerência foi significativamente maior nos quantis regionais a que nos quantis não regionais.

De acordo com a espacialização dos quantis regionais 0,90 a 0,99 estimados a partir do ajuste da distribuição de Wakeby, para um tempo de recorrência de 50 anos, a região composta pelas estações de Cascavel e Toledo apresentou os ventos mais severos do Paraná. Seguida pelas regiões Oeste (composta por estações próximas ao reservatório de Itaipu) e Central (composta por estações localizadas desde o Planalto de Curitiba até o Planalto de Guarapuava), nesta ordem. As regiões Norte (em virtude da estação de Paranavaí) e do Litoral apresentaram os mais baixos quantis 0,98 do Paraná.

De forma geral, os resultados deste estudo mostraram que, apesar da curta duração de séries de vento, e sua consequente incerteza, a aplicação de métodos bem aceitos de identificação de regiões homogêneas, de seleção e de ajuste da distribuição de frequência regional para a estimativa dos quantis produz resultados validados por todos os testes estatísticos descritos em Hosking em Wallis (1997) e aplicados neste estudo.

\section{Referências}

AN, Y.; PANDEY, A.M. Techincal note: A comparison of methods of extreme wind speed estimation. Journal of Wind Engineering and Industrial Aerodynamics, v. 93, n. 7, p. 535-545, 2005.

ASSOCIAÇÃO BRASILEIRA DE NORMAS TÉCNICAS (ABNT). NBR 5422: Projeto de linhas aéreas de transmissão de energia elétrica. 1985. 
COOK, N.J. The designer's guide to wind loading of building structures. Part 1: Background, Damage Survey, Wind Data and Structural Classification. Building Research Establishment, Garston, and Butterworths. 371 p., London, 1985.

HOSKING, J.R.M.; WALLIS, J.R. Regional frequency analysis: an approach based on L-moments. Cambridge University Press, Cambridge, 224 p., 1997.

HOSKING, J.R.M. Fortran routines for use with the method of L-moments. Research report RC 20525(90933) 8/5/96, revision 7/25/05. IBM Research Division, 33 p., 2005.
PALUTIKOF, J.P.; BRABSON, B.B.; LISTER, D.H.; ADCOCK, S.T. A review of methods to calculate extreme wind speeds. Journal of Applied Meteorology, v. 6, n. 2, p. 119-132, 1999.

SHAFER, M.A.; FIEBRICH, C.A.; ARNDT, D.S. Quality assurance procedures in the Oklahoma mesonetwork. Journal of Atmospheric and Oceanic Technology, v. 17, n. 4, p. 474494, 2000.

This is an Open Access article distributed under the terms of the Creative Commons Attribution License, which permits unrestricted use, distribution, and reproduction in any medium, provided the original work is properly cited. 